\title{
A Reinforced Endotracheal Tube Completely Severed by a Patient Bite and Lodged in the Right Main Bronchus
}

\author{
Susie Yoon ${ }^{1}$, Hyunjung $\mathrm{Choo}^{1}$, Se Eun $\mathrm{Kim}^{1}$, Heeyeon Kwon ${ }^{2}$, and Hannah Lee ${ }^{1}$ \\ 'Department of Anesthesiology and Pain Medicine, ${ }^{2}$ Department of Plastic and Reconstructive Surgery, Seoul National University Hospital, \\ Seoul National University College of Medicine, Seoul, Korea
}

Reinforced endotracheal tubes (ETTs) are designed to resist kinking or compression. However, these have a potential risk of being obstructed or severed by a patient's bite. We report a case in which a reinforced ETT was severed by tube-bite while the patient was in the prone position during an intensive care unit stay. Bronchoscopic evaluation showed that the severed distal part of the tube had lodged in the patient's right main bronchus, and it had to be surgically removed. The patency of reinforced ETTs should be carefully monitored in patients intubated in the prone position.

Key Words: airway obstruction; bronchoscopy; intubation, intratracheal; prone position.

Reinforced endotracheal tubes (ETTs) are designed to resist kinking or compression. These tubes are frequently used during facial surgeries and neurosurgeries, and in patients in a non-supine position during surgery [1]. However, they carry a potential risk of being near-fatally obstructed under external forces [2-4]. Here, we report an extremely rare case in which a reinforced ETT was severed by tube-bite and whose distal portion lodged in the right main bronchus.

\section{Case Report}

A 74-year-old woman (height, $156 \mathrm{~cm}$; weight, $63 \mathrm{~kg}$ ), with American Society of Anesthesiologists physical status grade II, was diagnosed with malignant fibrous histiocytoma in the left buttock, for which she underwent surgery and left gluteal flap closure. She had a medical history of hypertension and chronic renal failure. The surgery was performed under general anesthesia for a prolonged time ( $24 \mathrm{~h}$ and $55 \mathrm{~min}$ ), and the patient was intubated with a 7.0-mm-internaldiameter Mallinckrodt ${ }^{\mathrm{TM}}$ (Covidien, Dublin, Ireland) cuffed reinforced ETT. The patient was transferred to the intensive care unit (ICU) and remained intubated because of postoperative upper airway edema. An oral airway was inserted as a bite block to prevent collapse of the tube. She was kept in a prone position with the reinforced ETT because the gluteal

Received on May 13, 2016 Revised on June 28, 2016 Accepted on July 21, 2016

Correspondence to: Hannah Lee, Department of Anesthesiology and Pain Medicine, Seoul National University Hospital, Seoul National University College of Medicine, 101 Daehak-ro, Jongno-gu, Seoul 03080, Korea

Tel: +82-2-2072-3538, Fax: +82-2-747-5639, E-mail: closerthan@gmail.com

*No potential conflict of interest relevant to this article was reported.

(c) This is an Open Access article distributed under the terms of the Creative Commons Attribution Non-Commercial License (http://creativecommons.org/ licenses/by-nc/4.0/) which permits unrestricted non-commercial use, distribution, and reproduction in any medium, provided the original work is properly cited. Copyright (c) 2017 The Korean Society of Critical Care Medicine 
flap turned blue in the lateral decubitus position. During the ICU stay, she was sedated with dexmedetomidine and remifentanil. The infusion rate of the drugs was controlled on the basis of the level of sedation, with a goal of achieving Richmond Agitation Sedation Scale (RASS) level 2. Although she was weaned from the mechanical ventilator in a pressure support ventilation mode, she could not be extubated because she tested positive on the cuff-leak test and underwent two additional emergency surgeries - one on the same day and another $48 \mathrm{~h}$ later. On postoperative day 3 , she still tested positive on the cuff-leak test one and a half hours before the event. Also, the charge nurse had difficulties passing a suction catheter through the lumen of the ETT because of the narrow lumen, but the tidal volume and peak inspiratory pressure were not changed. Thirty minutes before the event, the tidal volume and peak inspiratory pressure were still unchanged. Peak airway pressure suddenly increased with a decrease in the tidal volume, and oxygen satura-

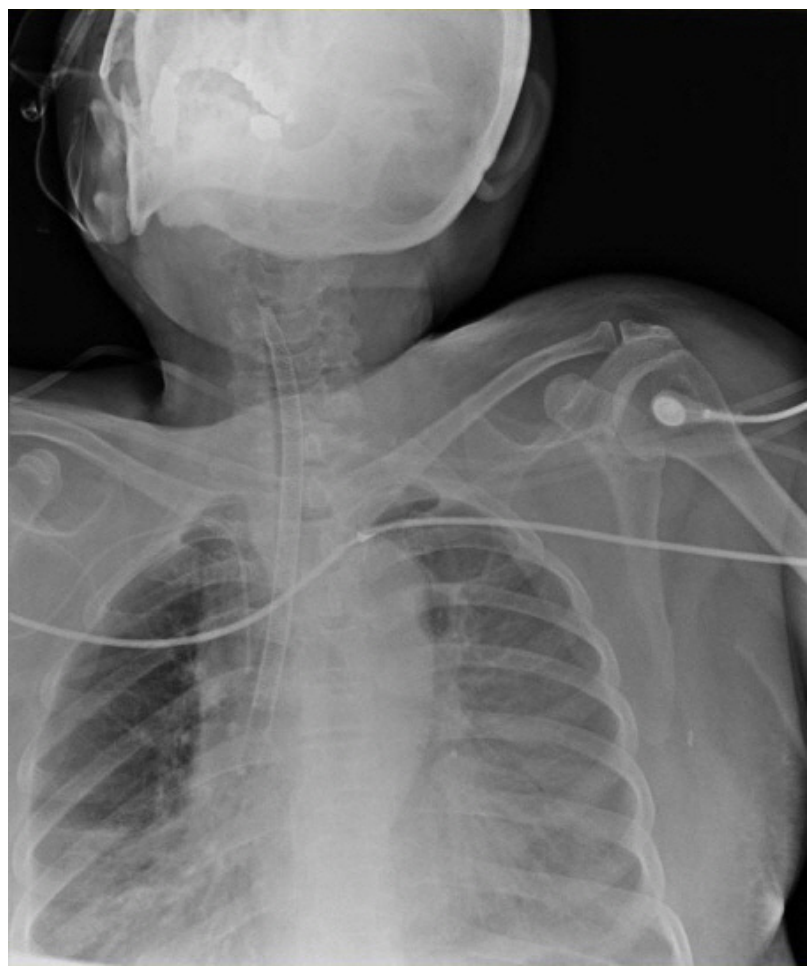

Figure 1. A chest anteroposterior radiograph showing the distal portion of the severed endotracheal tube lodged in the right main bronchus. tion was reduced to $85 \%$ despite the delivery of $100 \%$ oxygen. We attempted to pass a suction catheter through the lumen of the ETT, but the catheter was obstructed at the level of the incisor teeth. After placing the patient in a supine position, we found that the ETT had been completely severed and the oral airway had been spat out. Upon inspection of the oral cavity, we discovered that the distal portion of the ETT was missing. Therefore, we performed fiberoptic bronchoscopy after normalizing the oxygen saturation to $98-100 \%$ (flow rate, $10 \mathrm{~L} / \mathrm{min}$ ) by using a facial mask. Although the severed distal portion of the ETT was observed beneath the vocal cord, it could not be removed. Chest anteroposterior radiography showed that the severed portion was lodged in the right main bronchus (Figure 1). Her self-respiration was maintained without upper airway irritation during the time required for exams and the surgical procedure. The patient was taken to the operating room for emergency bronchoscopy. The ETT was surgically removed by an otolaryngologist under general anesthesia. In the operating room, she was preoxygenated with $100 \%$ oxygen, induced with intravenous propofol $1.5 \mathrm{mg} / \mathrm{kg}$ without the use of a muscle relaxant and anesthesia was maintained with manual ventilation with $100 \%$ oxygen and sevoflurane. After achieving adequate depth, a micro-laryngoscope was introduced and the severed ETT was removed with a forceps successfully (Figure 2). Micro-laryngoscopy revealed a mild subglottic swelling right after removal of the ETT. As the patient recovered spontaneous breathing,

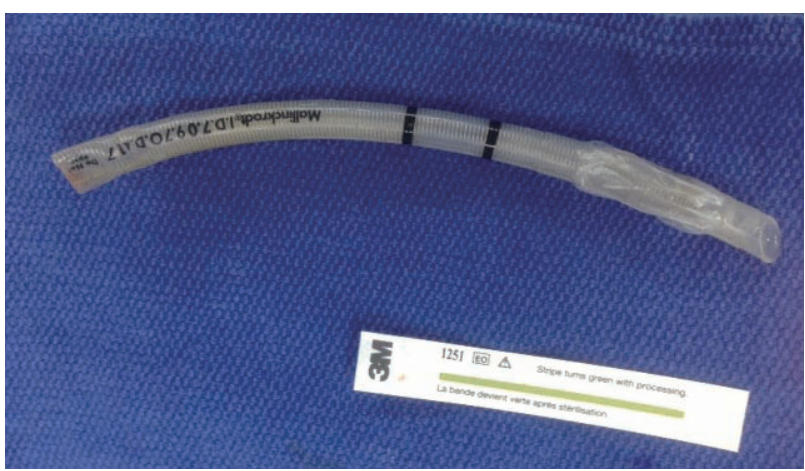

Figure 2. The distal portion of the endotracheal tube is completely severed by the patient's bite. 
she was transferred to the ICU. Additional intubation was not needed and $5 \mathrm{mg}$ dexamethasone was intravenously injected twice a day for 2 days for relieving upper airway edema. The remainder of the patient's ICU stay was uneventful, and she was transferred to a ward without any difficulty with respiration. Further laryngeal evaluation showed no abnormal findings. However, the gluteal flap did not remain viable and underwent necrosis; hence, the patient had to undergo another surgery for flap removal. She was then transferred to the regional hospital for palliative care.

\section{Discussion}

Our case report presents a very rare complication involving the use of reinforced ETTs. The most commonly reported major complication involving the use of reinforced ETTs is obstruction usually caused by bubble formation along the tube walls when using $\mathrm{N}_{2} \mathrm{O}$ gas $[5,6]$, foreign bodies, and tube-bites [7,8]. A tube-bite could result in deformity of the embedded wires, irreversible obstruction, and even excision of the tube. This may happen when the depth of anesthesia is inadequate or during recovery from anesthesia [7,8]. A tube-bite resulting in a severed ETT in the prone position has been seen during surgery [9], but it has never been reported in an ICU. To our knowledge, this is the first case in which a reinforced ETT was completely severed and the severed part lodged in the right main bronchus of the patient during the ICU stay.

Owing to its flexible and kink-resistant design, a reinforced ETT is useful during surgery, especially when the patient is in a prone position. Occasionally, these patients require postoperative mechanical ventilation and intensive care. In such instances, it is advisable to replace the reinforced ETT with a conventional tube. This will prevent obstruction due to tube-biting that might occur when the patient emerges from anesthesia [3]. In the present case, the tube was not replaced because the patient had severe upper airway edema and had to maintain a prone position during postoperative transfer to the ICU as well as during the ICU stay.

Ventilatory distress in a prone position may be a lifethreatening complication. Our patient was sedated with remifentanil and dexmedetomidine without any muscle relaxant to preserve self-respiration. We controlled the rate of continuous infusion of sedatives and analgesics based on the patient's pain response and agitation according to the critical care non-verbal pain scale and RASS. However, the oral airway was not securely fixed because of oral secretion; this, in turn, led to the patient spitting out the oral airway and biting the tube under light sedation. Therefore, proper bite blocks should be used with reinforced ETTs, and daily assessments should be conducted to determine readiness for extubation. Moreover, medical personnel in the ICU must be aware of the complications associated with reinforced ETTs. When such complications occur, the accepted procedure is to place the patient in a lateral or supine position immediately and to evaluate ETT patency with a $100 \%$ oxygen supply.

In conclusion, ETTs, which were developed to maintain airway patency, may compromise airway patency and can obstruct the respiratory tract as a foreign body. Medical personnel should pay attention to the patency of a reinforced ETT, especially in patients on a mechanical ventilator in a prone position.

\section{ORCID}

Susie Yoon http://orcid.org/0000-0001-5281-5904

Hannah Lee http://orcid.org/0000-0002-4001-1826

\section{References}

1. Haas CF, Eakin RM, Konkle MA, Blank R. Endotracheal tubes: old and new. Respir Care 2014; 59: 93352; discussion 952-5.

2. Mercanoglu E, Topuz D, Kaya N. The dissection of reinforced endotracheal tube internal wall causing intraoperative airway obstruction under general 
anesthesia: case report. Braz J Anesthesiol 2013; 63: $372-4$.

3. Brusco L Jr, Weissman C. Pharyngeal obstruction of a reinforced orotracheal tube. Anesth Analg 1993; 76: 653-4.

4. Ball JE, Platt S. Obstruction of a reinforced oral tracheal tube. Br J Anaesth 2010; 105: 699-700.

5. Populaire C, Robard S, Souron R. An armoured endotracheal tube obstruction in a child. Can J Anaesth 1989; 36(3 Pt 1): 331-2.

6. Santos IA, Oliveira CA, Ferreira L. Life-threatening ventilatory obstruction due to a defective tracheal tube during spinal surgery in the prone position. Anesthesiology 2005; 103: 214-5; discussion 215.

7. Azim A, Matreja P, Pandey C. Desaturation with flexometallic endotracheal tube during lumbar spine surgery-a case report. Indian J Anaesth 2003; 47: 48-9.

8. Balakrishna P, Shetty A, Bhat G, Raveendra U. Ventilatory obstruction from kinked armoured tube. Indian J Anaesth 2010; 54: 355-6.

9. Chalkeidis O, Kalakonas A, Chaidutis C, Chotoumanidis C. Endotracheal tube cutting during neurosurgical operation. Eur J Anaesthesiol 2009; 26: 533-4. 